\title{
Service Level Agreements: Web Services and Security ${ }^{\star}$
}

\author{
Ganna Frankova \\ Dept. of Information and Communication Technologies, Univ. of Trento, Trento, Italy \\ ganna.frankova@unitn.it \\ Advisor: Prof. Marco Aiello, Co-Advisor: Prof. Fabio Massacci
}

\begin{abstract}
To support the quality of service guarantee from the service provider side, complex web services require to be contracted through service level agreement. State of the art on web services and web service compositions provides for a number of models for describing quality of service for web services and their compositions, languages for specifying service level agreement in the web service context, and techniques for service level agreement negotiation and monitoring. However, there is no framework for service level agreement composition and composition monitoring, the existing design methodologies for web services do not address the issue of secure workflows development. The present research proposal aims to develop concepts and mechanisms for service level agreement composition and composition monitoring. A methodology that allows a business process designer to derive the skeleton of the concrete secure business processes from the early requirements analysis would benefit.
\end{abstract}

\section{Introduction}

The use of Web Services (WS) requires quality guarantee from the service provider. Taking into account that the guarantee depends on actual resource usage, the service client and provider must agree a priori by specifying an agreement. This allows the provider to allocate the necessary recourses to support the quality of service guarantees. Additionally, the guarantees on service quality must be monitored and the service client must be notified in case of failure to meet the guarantees.

The Service Level Agreement (SLA) opens a wide spectrum of challenges. In this research proposal we address some of them and propose building SLA framework for SLA composition and composition monitoring. The existing design methodologies for web services do not address the issue of developing secure web services, secure business processes and secure workflows. The present research proposal aims to develop a methodology that allows a business process designer to derive the skeleton of the concrete secure business processes from the early requirements analysis.

\footnotetext{
* This work has been partly supported by the IST-FP6-IP-SERENITY project.
} 
The work is structured as follows: Section 2 provides background and specifies the problem area. Section 3 is devoted to the research objectives and directions, i.e., the problem statement. The approaches and methods to be applied in order to achieve the objectives, the results obtained so far and a tentative plan for future work are presented in Section 4.

\section{Background and Problem Area}

With the term Quality of Service $(Q \circ S)$ we refer to non-functional properties of services. Although various approaches for modelling quality of service for web services have been addressed in a number of recent works, in [4] we claim that current models are far from ideal and there is a lot of space for further investigation and innovative research.

To optimally select component services for a quality-aware composite service building Zeng et al. 21] propose to use a global planning approach. In [13] Lin et al. doubt about the approach and propose a fuzzy way to express QoS requirements. The feasibility of using workflow patterns to determine the QoS in a web service composition is demonstrated by Jaeger et al. [8].

There are several proposals addressing the issue of web services design based on early requirements [12, 18, 9]. The design methodologies do not aim to design secure web services, secure business processes and workflows. Some approaches [5], 3] aim to design secure systems. On the negative site, the approaches do not support the design of software and business processes based on a service-oriented architecture.

Covering web services with SLA opens new research directions such as SLA specification and monitoring. Although such languages as SLAng [11], WSOL [19] and WSLA [15] for specifying SLA have been proposed, specification of SLA is still a research topic 20,16]. Fundamental concepts of non-functional SLA monitoring are presented in [17]. Web Service Level Agreement framework for defining and monitoring SLAs is presented in [10]. CREMONA, an architecture for creating and monitoring agreements in a context of web services is proposed in 14. But all the approaches do not support monitoring SLA with the goal of anticipating terms violations.

\section{Emergent Questions: SLA Composition and Monitoring}

The cornerstone of web services success lies in the ability to compose web services on the fly in order to build complex added-value services. Dealing with quality aware web service composition requires the study of a number of problems, namely:

1. definition of a quality of service model to provide quality of service information at the level of individual web services;

2. building complex web services with multiple QoS constraints;

3. ensuring that a promised quality of service is actually provided during execution; 
4. developing secure business processes and secure workflows in the web services context;

5. finding and monitoring global SLA of a composition of web services.

In 4, we have addressed the first and second issues. A solution to the third issue was proposed in [1] by using a methodology to handle changes during the interactions of web services and to prevent the violation of QoS constraints. In this work, we consider the above problems and focus on the fifth and fourth issue.

\section{Research Objectives and Directions}

\section{Problem Statement}

Covering web service aggregation with SLAs has opened a number of critical issues regarding SLA composition and monitoring, namely:

(1) global SLA for web service composition calculation

Assume that a SLA is defined for each web service from a set of $\mathrm{n}$ services, i.e., $S L A_{1}, S L A_{2}, \ldots, S L A_{n}$. The services are used to build a web service composition (see the orchestration unit) and the client needs to know the global SLA of the whole composition, i.e., $S L A_{G}$. The scheme depicted on Figure 1 shows the corresponding scenario.

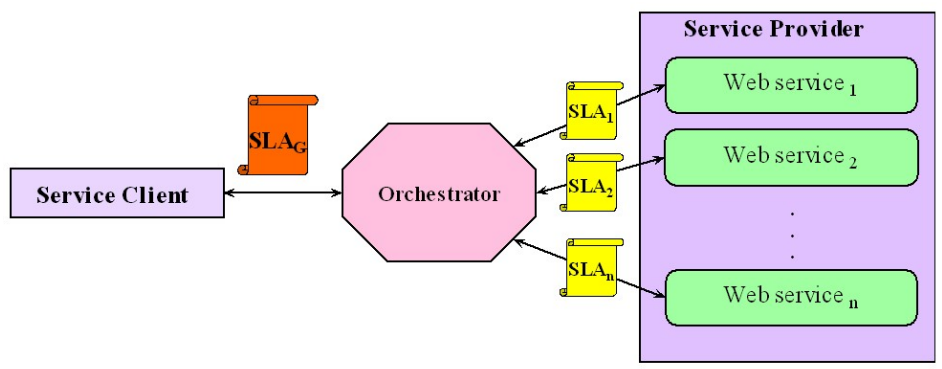

Fig. 1. "Service Client-Service Provider" interaction scheme

Depending on whether a goal of the clients is specified or not, one can consider two cases. Case 1: The client's goal is not specified. A global SLA $S L A_{G}$ of web service composition is calculated based on the SLAs $S L A_{i}$ coming from the individual web services $S_{i}, \mathrm{i}=1 . . \mathrm{n}$ to be composed. Calculating the global SLA should consider the business process $\operatorname{BP}\left(S_{1}, \ldots, S_{n}\right)$. Case 2: The client's goal is specified. Considering the specified $S L A_{G}$, such a set of web services $S_{i}$ and SLAs $S L A_{i}, \mathrm{i}=1$..n covering the web services are received that the business process $\operatorname{BP}\left(S_{1}, \ldots, S_{n}\right)$ guarantees the specified goal. There is an optional input of a set of web services $S_{j}$ and SLAs $S L A_{j}, \mathrm{j}=1 . . \mathrm{m}, \mathrm{m} \leq \mathrm{n}$ covering the web services in the scenario. 
Our research will be focused on calculation of global SLA for web service composition. Technique for composing SLA development are seen as a challenge.

(2) SLA for web service composition monitoring

Usually SLA monitoring techniques consider guaranteeing the fulfillment of a service term. We claim that how the guarantee is fulfilled should be taken into account. The answer to the question "Is the guarantee close or far from being violated?" should be given by the SLA framework. Our SLA framework will provide monitoring based on the client's goal described in SLA. It will not only predict and notify terms violations, but also discover the components of the composition that are responsible for the violation.

(3) secure workflow design based on early requirements

Requirements engineering has little counterpart in the development of business processes for web services. The existing design methodologies for web services do not address the issue of developing secure web services, secure business processes and secure workflows. This part of the work is devoted designing of secure business processes and secure workflows for web services. We aim to develop a methodology that allows a business process designer to derive the skeleton of the concrete secure business processes from the early requirements analysis.

\section{Approaches and Methods}

In order to realize the research objectives, a number of issues has been addressed and the results that fit in the framework of the thesis are obtained, namely:

\section{SLA Analysis}

The WS-Agreement [6] protocol has been studied and the earlier missed formal definition of SLA in a context of web services has been provided. The proposed set of formal rules ties together agreement terms and the life-cycle of an agreement. From the analysis some shortcomings of the protocol have been discovered. In particular, (i) there is no checking of how close a term to be violated and (ii) breaking one term of agreement results in terminating the whole agreement, while more flexibility is needed. The possible evaluations of agreements have been found and ways in which to make an agreement more robust and long lived have been identified. Two extensions to the specification and supporting environment have been proposed. The first is used to anticipate violations, while the second is devoted to run-time renegotiation.

\section{SLA Composition}

In the proposed methodology, we use hypergraphs [2] to capture the structure of business process. We see the problem of finding the global SLA of a business process as the Business Process Hypergraph (BPH) decomposition path evaluation. Suppose that a BPH is a weighted hypergraph. Each decomposition arc is associated a set of weights that show contribution of a source node to the target one. Each leaf node is assigned with a QoS value that corresponds to the QoS of atomic service. Each decomposition arc is assigned with an aggregation function which calculates the value of a target node taking as arguments 
source nodes and the set of weights. In order to evaluate a $\mathrm{BPH}$ decomposition path, one has to design the aggregation functions. In our approach the design of the aggregation functions depends on the structural activity that represent each compound service in BPH. The structural activity represents the basic structural elements of a composition as sequence, parallel execution, switch. Then, it is possible to perform the aggregation of numerical QoS dimensions in the spirit of Jaeger et. al [8]. The global SLA of a business process is the cost of decomposition path of BPH. As an extension of the suggested methodology, we propose to find the "minimal" decomposition path leading to the global SLA of the business process fulfilment. At the current moment we are finalizing the methodology and working with an industrial partner on the aggregation functions design and the mathematic model justification from the business point of view.

\section{SLA Monitoring}

The results of our work [1] will be used to build a monitoring system with anticipate terms violation goal. The proposed requirements for the rules specifying warning issuing are the following: easy to compute to avoid overloading of the monitoring system and be fast to provide warnings. In addition they should provide good performance in detecting as many violations as possible generating the minimum number of false positives. The linear least squares method was chosen as a forecasting method. We have conducted preliminary experimentation to show the feasibility of the anticipate violations strategy. In the experimentation based on synthetic data, more than $92 \%$ of violation points are warned in advance, and $96.5 \%$ of thrown warnings are true warnings.

\section{Secure Workflow Design}

We address the issue of secure workflows design based on early requirements analysis, namely, $S I^{*} /$ Secure Tropos [7, by presenting a methodology that bridges the gap between early requirements analysis and secure workflows for web services development. The methodology allows a business process designer to derive the skeleton of the concrete secure business processes from the early requirements analysis. The proposed refinement methodology, aims to obtain an appropriate coarse grained secure business process that can be further refined into workflows. We introduce a specification language for secure business processes Secure BPEL, which is a dialect of WS-BPEL for the functional parts and abstracts away low level implementation details from WS-Security and WSFederation specifications. At this point we have an open "lack of permission" problem. The "lack of permission" situation appears when there is a chain of delegation/trust of execution with no corresponding chain of delegation/trust of permission. In the Secure BPEL language both delegation and trust are modeled by invocation. In order to address the "lack of permission" problem one needs of introducing special types of invocation that allow the data to be protected, i.e, allows message confidentiality and integrity. Currently, we plan to extend the methodology to derive SLA from early requirements analysis. 


\section{References}

1. Aiello, M., Frankova, G., Malfatti, D.: Whats in an agreement? An analysis and an extension of WS-Agreement. In: Proceedings of the 3rd International Conference on Service-Oriented Computing (2005)

2. Ausiello, G., Italiano, G.F., Nanni, U.: Optimal traversal of directed hypergraphs. Technical Report TR-92-073 (1992)

3. Cheng, B.H.C., Konrad, S., Campbell, L.A., Wassermann, R.: Using Security Patterns to Model and Analyze Security Requirements. In: IEEE Workshop on Requirements for High Assurance Systems (2003)

4. Frankova, G.: Web service quality composition modelling. In: Proceedings of the $\mathrm{PhD}$ Symposium at 3rd International Conference on Service-Oriented Computing (2005)

5. Georg, G., Ray, I., France, R.: Using Aspects to Design a Secure System. In: Proceedings of IEEE International Conference on Engineering of Complex Computer Systems, Greenbelt, Maryland, USA (December 2 - 4, 2002)

6. GGF. Web Services Agreement Specification (WS-Agreement) (September 2005)

7. Giorgini, P., Massacci, F., Mylopoulos, J., Zannone, N.: Requirements Engineering for Trust Management: Model, Methodology, and Reasoning. International Journal of Information Security 5(4), 257-274 (2006)

8. Jaeger, M.C., Rojec-Goldmann, G., Mühl, G.: QoS Aggregation for Service Composition using Workflow Patterns. In: Proceedings of the 8th International Enterprise Distributed Object Computing Conference (2004)

9. Kazhamiakin, R., Pistore, M., Roveri, M.: A Framework for Integrating Business Processes and Business Requirements. In: Proceeding of the Enterprise Distributed Object Computing Conference (2004)

10. Keller, A., Ludwig, H.: Defining and monitoring Service Level Agreements for dynamic e-business. In: Proceedings of the 16th USENIX System Administration Conference (2002)

11. Lamanna, D.D., Skene, J., Emmerich, W.: SLAng: A language for defining Service Level Agreements. In: Proceedings of the 9th IEEE Workshop on Future Trends of Distributed Computing Systems (2003)

12. Lau, D., Mylopoulos, J.: Designing Web Services with Tropos. In: Proceedings of the IEEE International Conference on Web Services (2004)

13. Lin, M., Xie, J., Guo, H., Wang, H.: Solving QoS-driven web service dynamic composition as fuzzy constraint satisfaction. In: Proceedings of the IEEE International Conference on e-Technology, e-Commerce and e-Service (2005)

14. Ludwig, H., Dan, A., Kearney, R.: CREMONA: an architecture and library for creation and monitoring of WS-Agreements. In: Proceedings of the Second International Conference on Service-Oriented Computing (2004)

15. Ludwig, H., Keller, A., Dan, A., King, R.P., Franck, R.: Web Service Level Agreement (WSLA) language specification. Version 1.0. IBM Corporation (January 2003), http://www.research.ibm.com/wsla/

16. Molina-Jimenez, C., Pruyne, J., van Moorsel, A.: The role of agreements in IT management software. Architecting Dependable Systems III, pp. 36-58 (2005)

17. Molina-Jimenez, C., Shrivastava, S.K., Crowcroft, J., Gevros, P.: On the monitoring of contractual Service Level Agreements. In: Proceedings of the First IEEE International Workshop on Electronic Contracting (2004)

18. Penserini, L., Perini, A., Susi, A., Mylopoulos, J.: From Stakeholder Needs to Service Requirements. In: Proceeding of International Workshop on Service-Oriented Computing: Consequences for Engineering Requirements (2006) 
19. Tosic, V.: WSOL Version 1.2. Technical Report SCE-04-11, Department of Systems and Computer Engineering, Carleton University (July 2004)

20. Trienekens, J.J.M., Bouman, J.J., van der Zwan, M.: Specification of Service Level Agreements: Problems, principles and practices. Software Quality Journal 12(1), 43-57 (2004)

21. Zeng, L., Benatallah, B., Dumas, M., Kalagnanam, J., Sheng, Q.Z.: Quality driven web services composition. In: Proceedings of the 12th International conference on World Wide Web (2003) 